\title{
EFEITO DO TRATAMENTO FUNGICIDA E DA PROFUNDIDADE DE SEMEADURA NO CONTROLE DE BIPOLARIS SOROKINIANA EM SEMENTES DE TRIGO
}

\section{B.C. Barros; S.H. Furlan}

Instituto Biológico, Centro Experimental Central, CP 70, CEP 13100-970, Campinas, SP, Brasil. E-mail: bcbarros@biologico.sp.gov.br

\section{RESUMO}

\begin{abstract}
Através do tratamento de sementes de trigo avaliou-se o efeito de fungicidas no controle de Bipolaris sorokiniana em sementes semeadas em duas profundidades e na emergência e desenvolvimento das plântulas. Foram realizados testes em condições de laboratório e casa de vegetação, utilizando-se sementes da cultivar IAC 24, com níveis de incidência de 34,7\% do mencionado fungo. Todos os fungicidas reduziram a incidência de $B$. sorokiniana nas sementes de trigo sendo, inclusive, erradicado quando as sementes foram tratadas com guazatine, tebuconazole + imazalil e guazatine + imazalil. Não se evidenciou interação significativa entre tratamentos e profundidade de semeadura. Todavia, foi verificado retardamento na emergência e desenvolvimento das plântulas, principalmente nas fases iniciais, quando as sementes tratadas foram semeadas na maior profundidade. Observou-se alta correlação $\left(r=0,95^{* *}\right)$ entre incidência de B. sorokiniana nas sementes e severidade de sintomas na região da coroa das plãntulas desenvolvidas nos vasos e $r=-0,79^{*} e-0,75^{*}$ respectivamente, para as correlações entre incidência do patógeno e emergência, e incidência e altura de plantas.
\end{abstract}

PALAVRAS-CHAVE: Fungicidas, Triticum aestivum, Bipolaris sorokiniana.

\section{ABSTRACT}

EFFECT OF SEED TREATMENT AND DEPTH SOWING ON THE CONTROL OF BIPOLARIS SOROKINIANAI, IN WHEAT SEEDS. The efficiency of fungicides and depth of seed sowing on the control of Bipolaris sorokiniana in wheat seeds and on emergence and seedling development was evaluated. Lab and greenhouse tests were performed, using seeds of the IAC 24 wheat cultivar with $34.7 \%$ of natural infection. All chemical treatments reduced the incidence of B. sorokiniana, which was eliminated when the seeds were treated with guazatine, tebuconazole + imazalil and guazatine + imazalil. No significant interaction was seen between treatments and depth sowing. However, there was delaying of emergence and seedling development when the seeds treated were sown at greater depth, especially on the initial phases. There was observed a high correlation $\left(\mathrm{r}=0.95^{* *}\right)$ between percentages of wheat seed incidence by $B$. sorokiniana and seedling crown lesion severity, and $r=-0.79^{*}$ and $-0.75^{*}$, respectively for seed pathogen incidence and emergence and seed pathogen incidence and plant height in pots.

KEY WORDS: fungicides, Triticum aestivum, Bipolaris sorokiniana.

\section{INTRODUÇÃO}

A mancha marrom das folhas, ou helmintosporiose, e a podridão comum de raízes causadas por Bipolaris sorokiniana são doenças da cultura do trigo, que podem reduzir drasticamente a qualidade e o rendimento de grãos.

O patógeno está freqüentemente presente nas sementes, sendo estas importantes meios de disseminação e causa de epidemias nas regiões produtoras do
Brasil (REIS, 1982; Goulart et al., 1990), especialmente do Estado de São Paulo (LASCA et al., 1983; V EсHIATOet al., 1987). Além das sementes, os restos culturais, antes de serem decompostos no solo, servem como substrato à sobrevivência e multiplicação do agente causador da podridão radiculare de manchas foliares, sendo a prática da rotação de culturas o método mais eficiente e econômico para romper o seu ciclo biológico (ReIs et al., 1988). Todavia, a associação patógenosemente é um mecanismo eficiente de sobrevivência,

*Significativo no nível de $5 \%$ de probabilidade.

**Significativo no nível de $1 \%$ de probabilidade. 
pois o patógeno permanece em contato direto com seu hospedeiro mesmo em condições de armazenamento, o que facilita seu desenvolvimento nas novas plantas. Daí, a importância epidemiológica de sementes infectadas e a importância do uso de sementes sadias, ou do seu tratamento, como práticas associadas à rotação de cultura para reduzir a níveis mais baixos a taxa de transmissão desse fungo (REIS; ForCELINI, 1993; ReIs et al., 2001).

A maioria das cultivares de trigo empregadas hoje no Estado de São Paulo, e mesmo no país, não apresenta bom nível de resistência às manchas foliares, sendo muito dependente das condições climáticas (BARros, 1981, 1988; BARros et al., 2006).

É freqüente verificar-se elevada incidência de $B$. sorokiniana em sementes de trigo, podendo causar decréscimo na germinação das plantas e enfraquecimento e até morte de plântulas (LASCA et al., 1983; WiEse, 1977). Portanto, trata-se de uma doença importante que não se tem alcançado cultivares com bons níveis de resistência e as sementes são eficientes fontes de inóculo primário encontradas, ao longo dos tempos, nas nossas condições, com muita freqüência eem altos níveis de infecção(LASCA etal., 1983; VECHIATO et al.,1987).

O uso de sementes de boa qualidade ou tratadas é de fundamental importância, pois está diretamente relacionado com o estabelecimento da cultura e, conseqüentemente, com a produtividade. Tratamento de sementes com fungicidas já é uma tecnologia amplamente difundida e visa eliminar os fungos veiculados pela semente e protegê-las de fungos presentes no solo, por ocasião do início do desenvolvimento da cultura, garantindo o estabelecimento ideal da população de plantas, o que é essencial para o sucesso do cultivo. Em condições desfavoráveis à germinação e emergência das sementes, especialmente em condições de déficit hídrico (LASCA et al., 1983; CARESATo; BARRos, 1990) que tornam esses processos mais lentos, fazem com que as sementes fiquem por um período maior de tempo expostas a fungos presentes nas sementes e no solo, como B. sorokiniana, Alternaria tenuis, Fusarium spp., Rhizoctonia solani, entre outros. Por outro lado, certos produtos podem interferir no desenvolvimento das plantas sendo também mais perceptíveis nas condições de estresse (GOULART,1988; LASCA et al., 1983).

Muitas vezes, mesmo sem apresentar sintomas externos, as sementes podem estar infectadas por organismos agentes causais de doenças. Para se evitar a reintrodução na lavoura, de fungos patogênicos, como B. sorokiniana, a Comissão Centro-Sul Brasileira de Pesquisa de Trigo (EMBRAPA, 2005) recomenda tratar as sementes com fungicidas principalmente quando estas apresentarem incidência entre 10 a $40 \%$ desse fungo. Não recomenda usar como sementes quando o nível for superior a $40 \%$.

Para o controle de B. sorokiniana tem sido recomendado o tratamento de sementes com carboxin + thiram, difenoconazole, triadimefon e triticonazole (EMBRAPA, 2005). Atualmente, são apenas esses os fungicidas registrados para o tratamento de sementes de trigo (ANDREI, 1999).

Este trabalho teve por objetivo avaliar fungicidas no tratamento de sementes de trigo, semeadas em duas profundidades, quanto ao controle de $B$. sorokiniana, e sua relação com a emergência e desenvolvimento das plantas.

\section{MATERIAL E MÉTODOS}

Os experimentos foram conduzidos em laboratório e casa de vegetação, no ano de 2004, usando-se a cultivar IAC 24, com 34,7\% de B. sorokiniana.

Em laboratório as sementes foram tratadas e os produtos e as respectivas doses encontram-se descritos na Tabela 1. Os tratamentos foram efetuados em sacos plásticos adicionando-se os fungicidas sobre $500 \mathrm{~g}$ de sementes e $5 \mathrm{~mL}$ de água e agitando-se por 4 minutos, verificando-se uma completa cobertura das sementes pelos produtos.

Tabela 1 - Especificação dos tratamentos utilizados em sementes de trigo (cv. IAC 24).

\begin{tabular}{lllll}
\hline $\mathrm{N}^{\mathrm{o}}$ & Produtos e Formulação & Técnico & $\begin{array}{l}\text { Dose g ia/ } \\
100 \mathrm{~kg} \mathrm{sem} .\end{array}$ & $\begin{array}{l}\text { Dose pc/ } \\
100 \mathrm{~kg} \mathrm{sem} .\end{array}$ \\
\hline $1-$ & Orius 6\% CE & tebuconazole & 4,8 & 80 \\
$2-$ & Panoctine CE & guazatine & 75 & 300 \\
$3-$ & Orius Combi 5 FS CE & tebuconazole + imazalil & $3+2$ & 80 \\
$4-$ & Spectro SC & difenoconazole & 30 & 200 \\
$5-$ & Baytan SC & triadimenol & 40,5 & 270 \\
$6-$ & Mirage 450 CE & prochloraz & 20 & 44,5 \\
$7-$ & Panoctine CE+ Magnate CE & guazatine +imazalil & $45+1,5$ & $180+60$ \\
$8-$ & Testemunha & - & - & - \\
\hline
\end{tabular}

CE: concentrado emulsionável; SC: solução concentrada; FS: para uso em semente;

i.a.: ingrediente ativo; p.c. : produto comercial 
Após o tratamento as sementes foram analisadas para sanidade utilizando-se o método de papel de filtro em gerbox, com congelamento, segundo LiMONARD (1966). O delineamento experimental foi inteiramente casualizado, com 8 tratamentos e 12 repetições. Cada parcela constituiu-se de 25 sementes dispostas em placa gerbox, contendo três folhas de papel de filtro esterilizadas e embebidas em água destilada, perfazendo um total de 300 sementes analisadas para cada tratamento. Após o plaqueamento, as sementes foram submetidas por 24 horas à temperatura de $-20^{\circ} \mathrm{C}$, em um "freezer", para inibir a germinação. A incubação das sementes foi realizada em estufa tipo BOD mantidas à temperatura de $21 \pm 2^{\circ} \mathrm{C}$, sob regime de luz branca, alternada com doze horas de escuro. Após cinco dias de incubação os fungos foram identificados, considerando a porcentagem de sementes $\operatorname{com} B$. sorokiniana e $A$. tenuis que foram os fungos mais freqüentemente encontrados.

Em casa de vegetação foram realizados os testes de emergência e desenvolvimento das plântulas. Após o tratamento, as sementes foram semeadas em vasos de $20 \mathrm{~cm}$ de diâmetro contendo $7 \mathrm{~kg}$ de solo, adubados na base de $2 \mathrm{~g} /$ vaso, com uma mistura de adubos da formula 4 - 14 - 8 correspondente a $30 \mathrm{~kg}$ de N, 60 de $\mathrm{P}_{2} \mathrm{O}_{5}$ e 20 de $\mathrm{K}_{2} \mathrm{O}$ por hectare, respectivamente. Foram realizadas regas diárias, sem encharcamento, durante o período do experimento. O delineamento estatístico empregado foi blocos ao acaso, analisados em esquema fatorial, com 8 tratamentos $X 3$ blocos $X 2$ profundidades de semeadura $(1 \mathrm{e} 4 \mathrm{~cm})$. Estas profundidades, obtidas adicionando-se camadas de 1 e $4 \mathrm{~cm}$ de solo, foram escolhidas por se tratar de experimento em casa de vegetação para simulação de condição de campo, visto que, a campo, a recomendação da profundidade de semeadura varia de 2 a $5 \mathrm{~cm}$, conforme as condições do solo e umidade (EMBRAPA, 2005). Cada parcela foi composta por um vaso com 25 sementes. Fez-se a contagem das plântulas emergidas eas determinações de sua altura aos 7, 14, 21 e 30 dias após a semeadura(DAS). Trinta dias após a emergência foram arrancadas 15 plântulas de cada vaso e, após lavagem, avaliou-se o sistema radicular, compreendendo colo e sistema radicular, considerando-se os seguintes parâmetros: grau de infecção da região basal das plântulas, com base na extensão do sintoma de escurecimento em raiz inclusive lesões acima da coroa, conforme DieHL (1982). Consistiu em classificar em 4 categorias as intensidades dos sintomas observados sobre a região basal: plântulas sadias $=$ sem sintomas visíveis; infecções leves $=1-25 \%$ da área; infecções moderadas $=25-50 \%$; e infecções severas $=$ $>50 \%$, atribuindo-se valores $0,2,5$ e 10 para as quatro categorias de sintomas, respectivamente, calculandose finalmente a porcentagem ponderada da intensidade de doença, ou seja, o grau de infecção, pela fórmula $\mathrm{GI} \%=\sum$ (valor da categoria $\times \mathrm{n}^{\circ}$ de plantas na categoria)/total de plantas $x$ valor máximo das categorias) x 100. Também, da região basal das plântulas com sintomas, foram efetuados isolamentos para confirmação da presença do patógeno.

Os dados obtidos nos experimentos foram submetidos à análise de variância e na comparação das médias considerou-se o teste de Tukey a $5 \%$ de probabilidade. As análises foram feitas a partir dos dados originais, sem transformação, à exceção das porcentagens de emergência e de intensidade de sintomas (GI) que foram transformadas em $\sqrt{x+0,5}$. Foram realizados testes de correlação entre os dados de infecção das sementes por B. sorokiniana e de intensidade de sintomas na base das plântulas, de emergência e altura das plântulas.

\section{RESULTADOS E DISCUSSÃO}

Diversos gêneros de fungos foram observados nas sementes, sobre papel de filtro, sendo com maior freqüência $B$. sorokiniana, apresentadona Tabela 2 . De modo geral, observou-se redução da porcentagem de infecção das sementes tratadas em relação à testemunha com um comportamento semelhante entre os fungicidas, destacando-se o guazatine, tebuconazole + imazalil e guazatine + imazalil como os mais eficientes, erradicando $B$. sorokiniana das sementes.

Diversos trabalhos mostram a eficiência de fungicidas no tratamento de sementes de trigo, visando o controle de patógenos do trigo. Carboxin + thiram, guazatine + imazalil mostraram-se eficientes no controle de B.sorokiniana nas sementes segundoGOULART; PaIVA (1993). "In vitro", verificou-se que o fungo foi sensível aos produtos nuarimol, triadimenol, guazatine, que reduziram significativamente o crescimento micelial a partir de 1 ppm sendo que o guazatine, iprodione, procimidone e propineb reduziram mais eficientemente a esporulação do patógeno (BARROS; MARIOTTO, 1986).

Em trabalho desenvolvido por LASCA et al. (1984), todos os produtos analisados reduziram significativamente o nível de infecção das sementes sobressaindo-se iprodione e imazalil, com reflexo altamente positivo, no caso do imazalil, para a emergência das plantas e com menor ataque do patógeno em pósemergência.

A eficiência de guazatine + imazalil foi verificada no controle deB. oryzae (VALARINIet al.,1988). Também em trabalhos realizados por LASCA et al. (1984), LASCA et al. (1985) eANJOS; NASSER(1987), guazatine + imazalil foi um dos tratamentos que eliminou completamente o fungo das sementes de trigo. Goulart; PAiva (1991, 1993) também verificaram um efetivo controle desse patógeno com a mistura de guazatine + imazalil. 
Neste trabalho, a ação do fungicida guazatine sobre B. sorokiniana foi bastante evidenciada, com efeito ainda mais positivo quando associado ao imazalil, com reflexo inclusive na sanidade do sistema radicular das plântulas (Tabela 2).

Tabela 2 - Porcentagens de sementes de trigo (cv.IAC 24), infectadas com Bipolaris sorokinianaanalisadas pelo "blotter test", em função dos diferentes tratamentos de sementes e grau de infecção (GI) por B. sorokiniana em plântulas, em casa de vegetação.

\begin{tabular}{lll}
\hline Tratamentos & B. sorokiniana $(\%)$ & GI $(\%)$ \\
\hline Tebuconazole & $11,7 \mathrm{~b}$ & $1,9 \mathrm{~b}$ \\
Guazatine & $0 \mathrm{~d}$ & $1,3 \mathrm{~b}$ \\
Tebuc+Imazalil & $0 \mathrm{~d}$ & $0,7 \mathrm{~b}$ \\
Difenoconazole & $5,7 \mathrm{bc}$ & $2,9 \mathrm{~b}$ \\
Triadimenol & $5,3 \mathrm{bc}$ & $2,9 \mathrm{~b}$ \\
Prochloraz & $4,0 \mathrm{c}$ & $3,8 \mathrm{~b}$ \\
Guazatine+Imazalil & $0 \mathrm{~d}$ & $1,5 \mathrm{~b}$ \\
Test. & $34,7 \mathrm{a}$ & $17,0 \mathrm{a}$ \\
Semeadura $1 \mathrm{~cm}$ & - & $3,8 \mathrm{~A}$ \\
Semeadura $4 \mathrm{~cm}$ & - & $4,2 \mathrm{~A}$ \\
\hline CV $(\%)$ & 40,2 & 23,9 \\
\hline
\end{tabular}

Dados originais transformados para fins de análise estatística em $\sqrt{x+0,5}$.

A ação do tebuconazole foi incrementada pela adição de imazalil, verificando-se a ação mais importante do imazalil no controle do patógeno.

A eficiência demonstrada pelos tratamentos com tebuconazole, prochloraz, triadimenol e difenoconazole foi significativamente menor que dos demais fungicidas. Os resultados deste trabalho, embora relativos a patógeno do trigo, de certo modo, corroboram com os apresentados por CADORIN; REIS (2003) que verificaram que produtos como triadimenol e difenoconazole, não inibiram "in vitro" o desenvolvimento de Dreschlera teres e concluiram que não demonstram potencial para serem usados em tratamento de sementes de cevada visando ao controle daquele patógeno.

Também quanto ao prochloraz e tebuconazole, que apresentaram eficiência intermediária no controle de B. sorokiniana, resultados similares foram observados por LAsCa etal. (1983), LASCAetal. (1984), ForCELINI; Reis (1988) e Goulart; Paiva, (1993).

Todos os produtos reduziram o grau de infecção (GI\%), plântulas com sintomas, em relação ao tratamento controle. Com relação ao grau de infecção observado na região basal das plântulas arrancadas dos vasos, apresentado na Tabela 2 , verifica-se que as sementes não tratadas apresentaram maiores índices, o que corresponde aos resultados de análise de sanidade em laboratório. Entretanto, os demais tratamentosforam praticamente semelhantes entresi, também demonstrando alguma eficiência sobre $B$. sorokiniana. Mesmo com relação às diferentes profundidades de semeadura, não se observou diferença significativa quanto à manifestação dos sintomas (GI) na base das plântulas em vasos.

Nosisolamentos realizados a partir das plântulas, comsintomas de escurecimento/podridão basal, sempre foi confirmada a presença de $B$. sorokiniana, enquanto que $A$. tenuis em muito pequena freqüência. Isto pode mostrar a maior importância do primeiro fungo como patógeno.

A análise de variância demonstrou não haver interação significativa entre profundidade de semeadura e tratamentos para quaisquer dos fatores analisados.

Tabela 3 - Porcentagens de plântulas de trigo emergidas em vasos com solo e altura das mesmas obtidas aos 7, 14, 21 e30 dias após a semeadura (DAS), referente aos diferentes tratamentos de sementes e médias, em função da profundidade de semeadura.

\begin{tabular}{|c|c|c|c|c|c|c|c|}
\hline \multirow[t]{2}{*}{ Tratamentos } & \multicolumn{3}{|c|}{ Emergência (\%) } & \multicolumn{4}{|c|}{ Altura $(\mathrm{cm})$} \\
\hline & 7 DAS & 14 DAS & $\overline{21 \mathrm{DAS}}$ & 7 DAS & 14 DAS & $21 \mathrm{DAS}$ & $30 \mathrm{DAS}$ \\
\hline Tebuconazole & $70,7 \mathrm{a}$ & $81,3 \mathrm{a}$ & $82,0 \mathrm{~b}$ & $1,8 \mathrm{abc}$ & $12,2 \mathrm{bc}$ & $26,3 \mathrm{a}$ & $\overline{56,3 \mathrm{bc}}$ \\
\hline Guazatine & $78,7 \mathrm{a}$ & $87,3 \mathrm{a}$ & $88,0 \mathrm{ab}$ & $1,6 \mathrm{bc}$ & $13,2 \mathrm{bc}$ & $27,0 \mathrm{a}$ & $63,3 \mathrm{a}$ \\
\hline Tebuc+Imazalil & $64,7 \mathrm{a}$ & $84,0 \mathrm{a}$ & $86,0 \mathrm{ab}$ & $1,3 \mathrm{c}$ & $12,3 \mathrm{bc}$ & $26,2 \mathrm{a}$ & $56,8 \mathrm{abc}$ \\
\hline Difenoconazole & $80,7 \mathrm{a}$ & $87,3 \mathrm{a}$ & $87,3 \mathrm{ab}$ & $2,4 \mathrm{a}$ & $14,0 \mathrm{a}$ & 26,8 a & $59,8 \mathrm{ab}$ \\
\hline Triadimenol & $65,3 \mathrm{a}$ & $84,0 \mathrm{a}$ & $84,7 \mathrm{ab}$ & $1,2 \mathrm{c}$ & $11,5 \mathrm{c}$ & $27,2 \mathrm{a}$ & $57,9 \mathrm{abc}$ \\
\hline Prochloraz & $64,7 \mathrm{a}$ & $89,3 \mathrm{a}$ & $90,0 \mathrm{a}$ & $1,3 \mathrm{c}$ & $13,2 \mathrm{ab}$ & 26,8 a & $59,6 \mathrm{ab}$ \\
\hline Guazatine+Imazalil & $71,3 \mathrm{a}$ & $89,3 \mathrm{a}$ & $90,7 \mathrm{a}$ & $1,1 \mathrm{c}$ & $12,3 \mathrm{bc}$ & $26,8 \mathrm{a}$ & 57,6 abc \\
\hline Testemunha. & $79,3 \mathrm{a}$ & 83,3 a & $84,7 \mathrm{ab}$ & $2,2 \mathrm{ab}$ & $12,2 \mathrm{bc}$ & $24,2 \mathrm{~b}$ & $52,5 \mathrm{c}$ \\
\hline Semeadura $1 \mathrm{~cm}$ & $81,5 \mathrm{~A}$ & $89,8 \mathrm{~A}$ & $90,8 \mathrm{~A}$ & $2,3 \mathrm{~A}$ & $13,4 \mathrm{~A}$ & $26,9 \mathrm{~A}$ & $58,4 \mathrm{~A}$ \\
\hline Semeadura $4 \mathrm{~cm}$ & $62,3 \mathrm{~B}$ & 81,7 B & $82,5 \mathrm{~B}$ & $0,9 \mathrm{~B}$ & $11,8 \mathrm{~B}$ & $25,9 \mathrm{~B}$ & $57,4 \mathrm{~A}$ \\
\hline$\overline{\mathrm{CV}(\%)}$ & 14,2 & 5,1 & 4,9 & 24,7 & 5,6 & 3,2 & 5,7 \\
\hline
\end{tabular}


Tabela 4 - Coeficientes de correlação simples obtidos entre os parâmetros avaliados em condições de laboratório e casa de vegetação.

\begin{tabular}{lcccc}
\hline & $\begin{array}{c}\text { Incidência de } B . \\
\text { sorokiniana nas } \\
\text { sementes }\end{array}$ & $\begin{array}{c}\text { Emergência } \\
\text { em solo }\end{array}$ & $\begin{array}{c}\text { Severidade de } \\
\text { infecção nas } \\
\text { plantas }\end{array}$ & Altura de plantas \\
\hline Incidência de B. sorokiniana nas sementes & 1,00 & $-0,79^{*}$ & $0,95^{* *}$ & $-0,75^{*}$ \\
Emergência em solo & & 1,00 & $-0,79^{*}$ & $0,74^{*}$ \\
Severidade de infecção nas plantas & & & 1,00 & $-0,67^{*}$ \\
Altura de plantas & & & 1,00 \\
\hline
\end{tabular}

*Significativo no nível de $5 \%$ de probabilidade.

**Significativo no nível de $1 \%$ de probabilidade.

A Tabela 3 mostra os dados da emergência das plantas em vaso, sob condições de casa de vegetação, nos intervalos de 7, 14 e 21 dias após a semeadura.

Neste trabalho, não se detectou diferença significativa na emergência das plântulas, todavia os menores valores absolutos, aos 7 DAS, foram obtidos com tratamentos com prochloraz, triadimenol e tebuconazole + imazalil. Verifica-se que a emergência de plântulas obtida sete dias após a semeadura variou de $64,7 \%$ (tebuconazole + imazalil; triadimenol; prochloraz) a 79,3\% (testemunha), porém não representou diferença estatística entre os tratamentos. Notase, na primeira avaliação, que apenas os tratamentos com tebuconazole+imazalil, prochlorazetriadimenol apresentaram os menores valores percentuais de emergência, sem, contudo, diferir estatisticamente dos demais tratamentos e da testemunha. Entretanto, já nas avaliações seguintes, aos 14 e 21 dias, observouse uma recuperação nas emergências e conseqüentemente uma menor diferenciação entre os tratamentos, demonstrando que os produtos, nessas condições, não interferiram significativamente na emergência das plantas.

A análise de correlação das médias evidenciou valores para $\mathrm{r}$ de $-0,79^{* *}$ e $0,95^{* *}$, para correlações entre infecção de sementes por $B$. sorokiniana e emergência das plantas, aos 14 dias, e severidade das lesões na região da coroa da planta, com possível influência negativa no estabelecimento da cultura.

Coeficientes de correlação simples, significativos nonível de $1 \%$ também foram obtidos entre os parâmetros analisados (Tabela 4). Concluiu-se que o nível de incidência de $B$. sorokiniana nas sementes, avaliado em laboratório, teve efeito sobre diversos parâmetros avaliados em condições casa de vegetação.

De maneira geral, não se verificou interferência negativa dos tratamentos sobre altura das plântulas, ocorrendo sim um efeito positivo, com o passar do tempo, obtendo-se valores superiores aos da testemunha, aos 21 dias após a emergência, evidenciando o resultado do controle do patógeno. Aos 30 dias, ou a partir daí, é possível que o crescimento das plantas tendama seequiparar em função da provável redução dos efeitos dos produtos.

Nos tratamentos com triadimenol, guazatine + imazalil e tebuconazole + imazalil, observou-se aos 7 DAS retardamento do desenvolvimento (Tabela 3), com menor altura das plântulas, fato este que nas avaliações posteriores (14, 21 e 30 DAS) não mais foi evidenciado, mostrando que em condições de umidade adequada houve recuperação do desenvolvimento.

Com relação à ação destes produtos químicos sobre o desenvolvimento das plantas, praticamente não se encontram relatos na literatura. Diversos trabalhos relatam essa forma de ação do triadimenol (GOULART, 1988) reduzindo o desenvolvimento inicial das plântulas, mas não se dispõe de informações, até então, do ocorrido também com guazatine + imazalil, tebuconazole + imazalil e prochloraz.

Estudos sobre tratamento de sementes de trigo demonstraram ser de grande valia a determinação prévia dos patógenos de potencial importância para a cultura, para indicação de produtos mais adequados. Os resultados obtidos no presente trabalho corroboram com outros que consideram importante a presença do fungo $B$. sorokiniana nas sementes de trigo e os possíveis efeitos inerentes aos fungicidas no estabelecimento da cultura.

\section{REFERÊNCIAS}

ANDREI, E. Compêndio de defensivos agrícolas. 6.ed. São Paulo: Andrei, 1999. 672p.

ANJOS, J.R.N.; NASSER, L.C.B. Eficiência de fungicidas para controle de Cochliobolus satious em sementes de

\footnotetext{
**Significativo no nível de $1 \%$ de probabilidade.
} 
trigo. In: RELATÓRIO TÉCNICO ANUAL DO CENTRO DE PESQUISA AGROPECUÁRIA DOS CERRADOS 1982/1985. Planaltina: EMBRAPA, 1987. p.446-449.

BARROS, B.C. Efeito do tratamento de semente de trigo (Triticum aestivum L.) com fungicidas sobre a germinação, emergência, desenvolvimento de doenças e rendimento. 1981. 59f. Dissertação (Mestrado em Fitopatologia) - Escola Superior de Agricultura "Luiz de Queiroz", Universidade de São Paulo, Piracicaba, 1981.

BARROS, B.C.; MARIOTTO, P.R. Ação de fungicidas sobre o desenvolvimento de Helminthosporium sativum Pamm., King \& Bakke "in vitro". Biológico, São Paulo, v.52, n.7/9, p.65-72, 1986.

BARROS, B.C. Avaliação da resistência varietal e da resposta de cultivares de trigo (Triticum aestivum L.) ao controle químico de oídio, mancha marrom e ferrugem da folha. 1988. 122f. Tese (Doutorado em Fitopatologia) - Escola Superior de Agricultura "Luiz de Queiroz", Universidade de São Paulo, Piracicaba, 1988.

BARROS, B.C.; CASTRO, J.L.; PATRÍCIO, F.R.A.

Resposta de cultivares de trigo (Triticum aestivum L.) ao controle químico das principais doenças fúngicas da cultura. Summa Phytopathologica, v.32, n.3, p.239-246, 2006.

CADORIN, A.L.R.; REIS, E.M. Erradication of Drechslera teres from barley seeds through fungicidal treatments. Summa Phytopathologica, v.29, n.2, p.199-201, 2003.

CARESATO, D.; BARROS, B.C. Efeito do controle de Pyricularia oryzae e Drechslera sorokiniana em trigo. Fitopatologia Brasileira, v. 2, n.15, p.134, 1990.

DIEHL, J.A.; TINLINE, R,D.; KOCHANN, R.A.; SHIPTON, P.J.; ROVIRA, A.D. The effect of fallow periods on common root rot of wheat in Rio Grande do Sul. Phytopathology, v.10, n.72, p.12971301, 1982.

EMBRAPA. Informações técnicas da Comissão Centro-Sul Brasileira de trigo e triticale para a safra de 2005. In: REUNIÃO DA COMISSÃO CENTRO-SUL BRASILEIRA DE PESQUISA DE TRIGO E TRITICALE, 20., 2005, Londrina, PR. Anais. Londrina: EMBRAPA, 2005. 234p.

FORCELINI, C.A.; REIS, E.M. Controle de Helminthosporium sativum, Septoria nodorum, Fusarium graminearum e Erysiphe graminis f.sp. tritici pelo tratamento de sementes de trigo com fungicidas. Fitopatologia Brasileira, v.13, n.1, p.31, 1988.

GOULART, A.C.P. Eficiência de três fungicidas no tratamento de sementes de trigo (Triticum aestivum) visando o controle do fungo Helminthosporium sativum P.K.\&B., em condições de laboratório. Revista Brasileira de Sementes, v.10, n.1, p.55-61, 1988.
GOULART, A.C.P.; MESQUITA, A.N.; PAIVA, F.A. Eficiência do tratamento químico de sementes de trigo no controle de Pyricularia oryzae e Cochliobolus sativus (Heminthosporium sativum). Fitopatologia Brasileira, v.15, n.4, p.302-305, 1990.

GOULART, A.C.P.; PAIVA, F.A. Controle de Pyricularia oryzae e Helminthosporium sativum pelo tratamento de sementes de trigo com fungicidas. Pesquisa Agropecuária Brasileira, v.6, n.11/12, p.1983-1988, 1991.

GOULART, A.C.P.; PAIVA, F.A. Eficiência do tratamento químico de sementes de trigo no controle de Helminthosporium satioum e Pyricularia oryzae. Summa Phytopathologica, v.19, n.3/4, p.199-203, 1993.

LASCA, C.C.; BARROS, B.C.; VALARINI, P.J. Levantamento de fungos de sementes de trigo produzidas no Estado de São Paulo e comparação de incidência da manchas foliares com infecção de sementes por Helminthosporium sativum. Summa Phytopathologica, v.9, p.95-96, 1983. Trabalho apresentado no CONGRESSO PAULISTA DE FITOPATOLOGIA, 6., 1983, Araras, SP, Resumos.

LASCA, C.C.; BARROS, B.C.; VALARINI, P.J.; FREGONEZI, L.E.; CHIBA, S. Eficiência de fungicidas em tratamento de sementes de trigo (Triticum aestivum L.) para controle de Helminthosporium sativum Pammel, King \& Bakke. Biológico, São Paulo, v.50, n.6, p.125-130, 1984.

LASCA, C.C.; BARROS, B.C.; VALARINI, P.J.; CASTRO, J.L.; CHIBA, S. Ação de fungicidas em tratamento de sementes de trigo (Triticum aestivum L.) no controle de Helminthosporium sativum Pammel, King e Bakke. Biológico, São Paulo, v.51, n.9, p.225-231, 1985.

LIMONARD, T. A modified blotter test for seed health. Netherlands Journal of Plant Pathology, v.72, p.319-321, 1966.

REIS, E.M. Sementes de trigo infectadas por Helminthosporium sativum: fonte de inóculo para podridão comum das raízes e seu controle pelo tratamento com fungicidas. Summa Phytopathologica, v.8, n.3/4, p.29-39,1982.

REIS, E.M.; FERNANDES, J.M.C.; PICININI, E.C. Estratégias para o controle de doenças do trigo. Passo Fundo: EMBRAPA-CNPT, 1988. 50 p. (Documentos)

REIS, E.M.; FORCELINI, C.A. Transmissão de Bipolaris sorokiniana de sementes para órgãos radiculares e aéreos do trigo. Fitopatologia Brasileira, v.18, n.1, p.76-81, 1993.

REIS, E.M.; TREZZI, R.; MEDEIROS, A.C. Diagnose, patometria e controle de doenças de cereais de inverno. Londrina: E.S. Comunicação, 2001. 94p.

VALARINI, P.J.; LASCA, C.C.; CHIBA, S. Eficiência de fungicidas em tratamento de sementes de arroz para 
controle de Helminthosporium oryzae. Pesquisa Agropecuária Brasileira, v.23, n.1, p.41-44, 1988.

VECHIATO, M.H.; LASCA, C.C.; VALARINI, P.J.

Sobrevivência do fungo Helminthosporium sativum em sementes de trigo (Triticum aestivum) armazenadas. Fitopatologia Brasileira, v.12, n.3, p.226-231, 1987.
WIESE, M.V. Compendium of wheat diseases. St. Paul: American Phytopatholgical Society, 1977. 106p.

Recebido em $1 / 3 / 07$

Aceito em 4/11/08 